\title{
Does Emotional Labor Mediate the Relationship Between Transformational Leadership Style and Service Recovery Performance
}

\author{
Farhan Khan \\ Lyallpur Business School, Government College University, Faisalabad, Pakistan. \\ Rufia Saeed* \\ Department of Commerce, Government College Women University, Faisalabad, Pakistan \\ Shehzad Mehmood \\ Lyallpur Business School, Government College University, Faisalabad, Pakistan. \\ Muhammad Irfan \\ Department of Management Sciences, Riphah International University, Faisalabad, Pakistan
}

\begin{abstract}
The research is financed by Asian Development Bank. No. 2006-A171(Sponsoring information)
Abstract

This study aims to analyze the mediating role of emotional labor (EL) on the relationship between transformational leadership (TL) and service recovery performance (SRP). For this purpose, the primary data are gathered from 200 respondents who are currently working in service jobs that basically include interaction of customers in Pakistan. Reliability test and Barron and Kenny (1986) method are used to analyze the mediating role of EL on the relationship between TL and SRP. Results of this study shows that TL is positively (negatively) related to deep acting (surface acting) emotional labor strategy. Moreover, deep acting (surface acting) is positively (negatively) linked with SRP. The study concludes that EL mediates the relationship between TL and SRP.
\end{abstract}

Keywords: Emotional Labor, Service Recovery Performance, Transformational Leadership

DOI: $10.7176 / \mathrm{JRDM} / 63-01$

Publication date:March $31^{\text {st }} 2020$

\section{Introduction}

Service performance of employees is an essential source of economic improvement of a service institution as it helps to extricate one service institution over its competitors. Moreover, service workers of service industry are predictable to deliver service recovery performance (SRP) that helps in solving a facility failure and to gratify their clients by meeting their prospects (Babkas et al., 2003; Guucheit et al., 2014). Examples of service letdowns are very common in the industry of hotels which requires SRP contain unapproachable places through check-in, provision of wrong bills, unhygienic foods etc. (Lee et al., 2011a). Numerous positive results are linked with SRP such as the satisfaction and dependability of clients, positive response of customers and expect to purchase (Haa \& Jung, 2009; Lio, 2007; Len 2010a). Because actual service performance is essential for the achievement of the commerce, previous research investigated different aspects that can improve SRP (Bashaff \& Allan, 2000; Masaud \& Hameiden, 2013). Though, the literature of SRP has investigated numerous specific and institutional backgrounds (Kem \& Uh, 2012; Len, 2010; Bashaff \& Allan, 2000) but no linkage has been found between leadership and SRP.

Mainly, this is a gap in the literature of SRP as research confirmed that the leadership styles and conducts are essential for overall performance of employees (Babakuseetal.,2003; Guchaitetaal.,2014). Moreover, research depicts that transformational leadership (TL) has direct influences on the performance of employees in the services perspective (Lae et al., 2011b; Leiao and Chuaneg, 2007). TL participate and inspire others to enhance the potentials and performance (Buss, 1985) that involve the performance of their service. Though TL has been connected with the performance of workers in facility job. The literature of TL also proposed the possible link between TL and SRP (Lin, 2010b; Punjeisre et al., 2013). However, this research is an attempt to investigate this relationship.

Meanwhile, TL is a conceivable distal precursor, the association between TL and SRP has not been investigated or analyzed. Actually, research elaborated that TLs induce emotions and request to others on an expressive smooth thus distressing the performance of an employee through emotions (Dasborugh, 2006; Dasbough \& Askanasy, 2002). Hence, the present study discourses this gap in the literature of SRP by investigating the emotional labor (EL) as a probable mediator on the relationship between TL and SRP. It is not only mandatory for service industry employees to achieve their essential job responsibilities, but it is also mandatory that they must also cope with their emotions by conveying positive emotions at work, and while relating with clients (Kim, 2008). 
This kind of emotional behavior, effort and control of feelings in reaction to institutional demands is regarded as EL (Lum \& Chen, 20122; Granday, 2000; Hoschchild, 1979). Hence, present study emphasizes on TL as a distal precursor of SRP through EL.

The study, therefore, aims to (i) "examine the influence of Transformational leadership on service recovery performance", (ii) "examine the influence of emotional labor on service recovery performance and the (iii) mediating effect of emotional labor between Transformational leadership and service recovery performance".

\section{Literature Review and Development of Hypothesis}

Luo et al (2019) empirically investigated the association between transformational leadership (TFL) and EL by using the sample of 217 workers of front-line hospitality of US and found the significant association between these two variables. Chuang, Judge \& Liaw (2012) had done a valuable work by examining the mediating effect of the emotional regulations of employees on the association between TFL and favorable customer intensions by using the data of 204 customers and service employees. the study found that emotional regulations mediated the relationship between TFL and favorable customer intensions. Grandy (2000) review the prevailing literature and suggested that workers are directed by TFL and they have faith on their managers, leaders and institutions so therefore they might choose deep acting while dealing their emotions.

After reviewing the above literature, we found different studies on the relationship between TFL and EL (Luo et al, 2019) We didn't find any study in which the relationship between TL and EL has been investigated. So therefore, there is a need to investigate the relationship between TL and EL because TLs induce emotions and request to others on an expressive smooth thus distressing the performance of an employee through emotions (Dasborugh, 2006; Dasbough \& Askanasy, 2002).

Luo et al (2019) examined the influence of EL on SRP by using two important dimensions of EL that are deep acting and surface acting. The study utilized the data of 217 front desk employees of US and showed that SRP is positively influenced by deep acting while surface active has negative effects on SRP. Lyubomirsky et al (2005) studied the impact of different emotional circumstances on the SRP and found that positive emotional condition has positive effects on SRP while negative emotional situations have negative effects on SRP. Huang \& Dai (2010) work on the relationship between emotions and SP of employees by using surface acting as a measurement of emotions of labor. The conclusions of this research showed the negative association between EL and SRP. Similarly, Kau \& Wan-Yiun Loh (2006) also showed the negative impacts of EL on SRP and concluded that honest terminologies of responsiveness, consideration, graciousness, and other positive emotions are the determinants of SRP.

Luo et al (2019) studies the effect of TFL on SRP. For this purpose, the data were gathered from two diverse cultures i.e. China and US. 217 front desk employees of US while 219 front desk employees of China were used as a sample of this study. The conclusions of this study revealed the positive impact of transformational leadership on SRP. The study also revealed the significant differences from these two cultures. Jiang, Zhao \& Ni (2017) examined the mediating impact of institutional residency performance on the relationship between TFL and SRP. For this purpose, a sample of 389 contractors were utilized. Result revealed that there is direct association between TFL and SRP. Moreover, the study also revealed that institutional residency performance mediates the relationship between TFL and SRP. Similarly, Liao \& Chuang (2007) also concluded the positive and significant relationship between TFL and SRP. Punjaisri, Evanschitzky \& Rudd (2013) studies the relationship between brand specific TFL and SRP. The study used corporate brand as a measurement of brand specific TFL and found that there is positive association between these two variables. Guchait, Simons \& Pasamehmetoglu (2016) investigated the impact of behavioral integrity of leader on SRP by collecting the data from the 369 employees of Turkish hotels. The findings of this study showed the direct relationship between these two variables

After reviewing the above literature, we found different studies on the relationship between TFL and SRP (Luo et al, 2019; Jiang, Zhao \& Ni, 2017; Liao \& Chuang, 2007; Punjaisri, Evanschitzky \& Rudd, 2013). We didn't find any study in which the relationship between TL and SRP has been investigated. So therefore, there is a need to investigate the relationship between TL and SRP because research depicts that transformational leadership (TL) has direct influences on the performance of employees in the services perspective (Lae et al., 2011b; Leiao and Chuaneg, 2007).

\subsection{Hypothesis Development}

H1. "TL is positively related to SRP."

$\mathrm{H} 2 \mathrm{a}$. "TL is positively related to DA".

H2b. "TL negatively related to SA".

H3a. "DA is positively related to SRP".

H3b. "SA is negatively related to SRP".

H4a. "DA mediates the relationship between TL and SRP".

H4b. "SA mediates the relationship between TL and SRP". 


\section{Data and Methodology}

The study is conducted on the mediating impact of EL on the association between TL and SRP. For this purpose, the primary data are gathered from 200 respondents that are working currently in-service jobs that basically involve interaction of customers in Pakistan. Reliability test and Barron and Kenny (1986) method (through regression analyses) are used to analyze the mediating impact of EL on the relationship between TL and SRP. Four types of variables are used in the study as provided in Table 1:

Table1: Description of Variables

\begin{tabular}{|l|l|}
\hline Type of Variable & Description \\
\hline Dependent & $\begin{array}{l}\text { Service Recovery Performance (SRP) } \\
\text { "SR is a company's resolution of a problem from a dissatisfied customer, converting } \\
\text { them into a loyal customer. It is the action a service provider takes in response to service } \\
\text { failure. The five-item measure by Boshoff and Allen (2000) was used to measure } \\
\text { service recovery performance using a 5-point Likert-type scale". }\end{array}$ \\
\hline $\begin{array}{l}\text { Independent } \\
\text { Variables }\end{array}$ & $\begin{array}{l}\text { Transformational Leadership (TL) } \\
\text { "TL is a leadership style where the executive relies on rewards and punishments to } \\
\text { achieve optimal job performance from his/her subordinates. As such, TL is based on an } \\
\text { exchange, or transaction: The leader rewards workers who perform their tasks to the } \\
\text { specified levels and punishes workers who do not perform to those set standards. The } \\
\text { measure of TL by Podsakoff et al. (1996) is used. This measure includes 23 items that } \\
\text { captures an overall index of TL using a 5-point Likert-type scale (Liao and Chuang, } \\
\text { 2007)". }\end{array}$ \\
\hline $\begin{array}{l}\text { Mediating } \\
\text { Variables }\end{array}$ & $\begin{array}{l}\text { Emotional Labor (EL) } \\
\text { "EL is the process of managing feelings and expressions to fulfil the emotional } \\
\text { requirements of a job. More specifically, workers are expected to regulate their } \\
\text { emotions during interactions with customers, co-workers and superiors. This includes } \\
\text { analysis and decision making in terms of the expression of emotion, whether actually } \\
\text { felt or not, as well as its opposite: the suppression of emotions that are felt but not } \\
\text { expressed. The study uses two measures of EL; deep acting and surface acting. Deep } \\
\text { acting (DA) is about a person trying to feel a specific emotion that they are thinking } \\
\text { about in their mind. Surface acting (SA) is when a person has to fake emotion to meet } \\
\text { certain social or work rules. The measure by Diefendorff et al. (2005) assessed surface } \\
\text { acting with seven items and deep acting with four items using a 5-point Likert type } \\
\text { scale". }\end{array}$ \\
\hline $\begin{array}{l}\text { Demographic Information } \\
\text { "Gender and age are used as control variables, because research shows that these } \\
\text { demographic variables can be related to emotional labour (Grandey, 2000). Thus, these } \\
\text { variables can potentially influence the results". }\end{array}$ \\
\hline Variables
\end{tabular}

\subsection{Survey Questionnaire}

The study has constructed a questionnaire (see appendix) comprising of 39 questions. The questionnaire is then divided in to 4 sections. The section 1 focuses on the respondents' basic information (age and gender). The section 2,3 and 4 comprises of items of SRP, TL and EL, respectively.

\subsection{Types of Questions and Likert Scale}

The study uses two types of question which are asked from the respondents. The first type of questions consists of multiple-choice questions which are used to analyze the basic information of the respondents. The second type comprises of liker type scale and covers the various aspects like SRP, TL and EL. These measurements are based on 5-points Likert scale.

\subsection{Method of Baron and Kenny (1986)}

The study uses the Barron and Kenny (1986) method to examined the mediating role of EL (DA and SA) on the association between TL and SRP. The study takes support from OLS regression for this purpose. Before testing the mediation, the assumptions of Baron and Kenny (1986) are satisfied. As per Barron and Kenny (1986), the association between explained and exploratory variable must be statically significant and also it is essential that the effect of exploratory variable on the mediator is statically significant. Correspondingly, the influence of mediating variable on explained variable must also be statistically significant. Once these assumptions are fulfilled, the study may proceed for mediation by using the method of Barron and Kenny (1986) 


\subsection{Econometric Model}

The following regression models are used in the study:

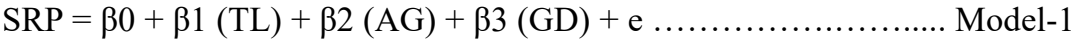

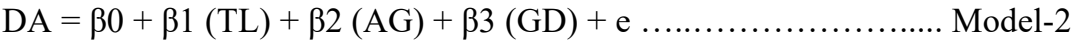

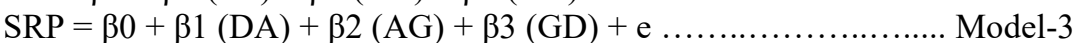

$\mathrm{SRP}=\beta 0+\beta 1(\mathrm{TL})+\beta 2(\mathrm{DA})+\beta 3(\mathrm{AG})+\beta 4(\mathrm{GD})+\mathrm{e} \ldots \ldots \ldots . . .$. Model -4

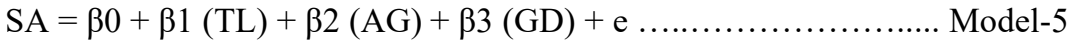

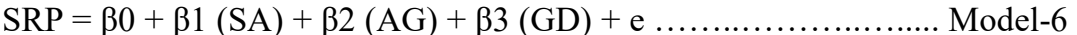

$\mathrm{SRP}=\beta 0+\beta 1(\mathrm{TL})+\beta 2(\mathrm{SA})+\beta 3(\mathrm{AG})+\beta 4(\mathrm{GD})+\mathrm{e} \ldots \ldots \ldots . . .$. Model -7

Where; "Surface acting (SA), deep acting (DA), transformational leadership (TL), service recovery performance (SRP), age (AG) and gender (GD). $\beta 0$ is constant, $\beta 1, \beta 2, \beta 3$ and $\beta 4$ are regression coefficient and e denote error term."

\section{Results and Discussions}

\subsection{Reliability of the Instruments}

Table 2 presents the estimated values of Cronbach's alpha coefficients to testing the reliability and internal uniformity of the measures. For the current sample, values of Cronbach's alpha vary from 0.77 to 0.89 indicating that each multi-item constructs preserves consistency. SRP $(\alpha=0.7731)$, TL $(\alpha=0.8945)$, SA $(\alpha=0.8479)$ and DA $(\alpha=0.8394)$. The values of Cronbach's alpha show that all constructs such as SRP, TL, SA and DA are highly reliable, internally consistent and the same content is universally measured by the item of each construct.

Table 2: Reliability Test

\begin{tabular}{|c|c|c|c|}
\hline Construct & Valid N & Number of Items & Cronbach's alpha \\
\hline SRP & 200 & 05 & 0.7731 \\
\hline TL & 200 & 23 & 0.8945 \\
\hline SA & 200 & 07 & 0.8473 \\
\hline DA & 200 & 04 & 0.8394 \\
\hline
\end{tabular}

Note: "According to Hinton et al. (2004), there are four cuts of points for reliability including excellent reliability ( 0.90 and above), high reliability $(0.70-0.90)$, moderate reliability $(0.50-0.70)$ and low reliability (0.50 or below)". "SRP: Service Recovery Performance, TL: Transformational Leadership, SA: Surface Acting, DA: Deep Acting."

\subsection{Descriptive Statistics}

Table 3 reveals the results of descriptive statistics of the variables. 6 variables are used in the study. Minimum, maximum, mean and standard deviation of data are shown in Table 3. SRP has the mean value of 0.0382 . The mean number of TL is 4.0021 . The average value of SA (DA) is 4.6312 (3.2641) while the mean value of AG (GD) is $2.6314(0.2381)$. SRP, TL, SA, DA AG and GD shows $\pm 53.24 \%, \pm 61.85 \%, \pm 60.84 \%, \pm 63.41 \%, \pm 53.14 \%$ and $\pm 43.25 \%$ variations, respectively.

Table 3: Descriptive Statistics

\begin{tabular}{|l|c|c|c|c|}
\hline Variables & Minimum & Maximum & Mean & Std. Deviation \\
\hline SRP & 2.0000 & 5.0000 & 3.0382 & 0.5324 \\
\hline TL & 1.0000 & 5.0000 & 4.0021 & 0.6185 \\
\hline SA & 1.0000 & 5.0000 & 4.6312 & 0.6084 \\
\hline DA & 1.0000 & 4.0000 & 3.2641 & 0.6341 \\
\hline AG & 1.0000 & 4.0000 & 2.6314 & 0.5314 \\
\hline GD & 0.0000 & 1.0000 & 0.2381 & 0.4325 \\
\hline
\end{tabular}

Note: "SRP: Service Recovery Performance, TL: Transformational Leadership, SA: Surface Acting, DA: Deep Acting, AG: Age, GD: Gender."

\subsection{Correlation}

Table 4 reveals the results of the correlation among the variables. The correlation is tested by using the Pearson's correlation matrix. The lowest coefficient of correlation is 0.1975 that is in between GD and DA. While the highest coefficient of correlation is 0.5214 that is in between AG and SA. It shows that multicollinearity does not affect the data. 
Table 4: Pearson Correlation Matrix

\begin{tabular}{|l|c|c|c|c|c|c|}
\hline Variables & SRP & TL & SA & DA & AG & GD \\
\hline SRP & 1 & & & & & \\
\hline TL & 0.2342 & 1 & & & & \\
\hline SA & 0.4315 & 0.5024 & 1 & & & \\
\hline DA & 0.2096 & 0.4823 & 0.3892 & 1 & & \\
\hline AG & 0.4932 & 0.2982 & 0.5214 & 0.3824 & 1 & \\
\hline GD & 0.3365 & 0.3128 & 0.3928 & 0.1975 & 0.4284 & 1 \\
\hline
\end{tabular}

Note: "SRP: Service Recovery Performance, TL: Transformational Leadership, SA: Surface Acting, DA: Deep Acting, AG: Age, GD: Gender."

\subsection{Mediation Analysis}

Table 5 shows the results of model 1-7 to analyze the mediation through Barren and Kenny (1986) method. Model1 shows that there is direct and significant association between TL and SRP which supports H1 of the study. From model-2, it can be seen that TL also has significant positive relationship with DA, supporting H2a. Model-3 reveals that there is significant positive association between DA and SRP, accepting H3a. Here, the assumptions of Barren and Kenny (1986) are fulfilled which allows the study to move to the model-4 to analyze the mediation. The model-4 shows that the coefficient of TL becomes insignificant which shows that DA mediates the relationship between TL and SRP. From model-5, it can be seen that TL has significant negative relationship with SA, supporting H2b. Model-6 reveals that there is significant positive association between SA and SRP, accepting H3b. Again, the assumptions of Barren and Kenny (1986) are fulfilled which allows the study to move to the model-7 to analyze the mediation. The model-7 shows that the coefficient of TL becomes insignificant which shows that SA mediates the relationship between TL and SRP.

Table 5: Regression Analysis

\begin{tabular}{|c|c|c|c|c|c|c|c|}
\hline Variables & $\begin{array}{c}\text { Model-1 } \\
\text { (SRP) }\end{array}$ & $\begin{array}{l}\text { Model-2 } \\
\text { (DA) }\end{array}$ & $\begin{array}{c}\text { Model-3 } \\
\text { (SRP) }\end{array}$ & $\begin{array}{c}\text { Model-4 } \\
\text { (SRP) }\end{array}$ & $\begin{array}{l}\text { Model-5 } \\
\text { (SA) }\end{array}$ & $\begin{array}{c}\text { Model-6 } \\
\text { (SRP) }\end{array}$ & $\begin{array}{c}\text { Model-7 } \\
\text { (SRP) }\end{array}$ \\
\hline \multirow{2}{*}{ TL } & 0.1551 & 0.2631 & & 0.0352 & -0.3412 & & 0.0314 \\
\hline & $\left(0.0002^{*}\right)$ & $\left(0.0000^{*}\right)$ & & $(0.2514)$ & $\left(0.0048^{*}\right)$ & & $(0.1463)$ \\
\hline \multirow{2}{*}{ DA } & & & 0.2962 & 0.0463 & & & \\
\hline & & & $\left(0.0027^{*}\right)$ & $(0.3514)$ & & & \\
\hline \multirow{2}{*}{ SA } & & & & & & 0.3931 & 0.2416 \\
\hline & & & & & & $\left(0.0004^{*}\right)$ & $(0.2145)$ \\
\hline \multirow{2}{*}{ AG } & 0.0236 & 0.3521 & 0.0236 & 0.6324 & 0.5214 & 0.0236 & 0.5214 \\
\hline & $(0.3812)$ & $(0.3824)$ & $(0.3812)$ & $(0.6521)$ & $(0.1934)$ & $(0.3812)$ & $(0.6324)$ \\
\hline \multirow{2}{*}{ GD } & 0.6314 & 0.4352 & 0.6314 & 0.4371 & 0.5899 & 0.6314 & 0.3742 \\
\hline & $(0.3412)$ & $(0.1421)$ & $(0.3412)$ & $(0.4215)$ & $(0.3715)$ & $(0.3412)$ & $(0.3718)$ \\
\hline
\end{tabular}

Note: “(*) Significant at 0.05, (p-values)”. "SRP: Service Recovery Performance, TL: Transformational Leadership, SA: Surface Acting, DA: Deep Acting, AG: Age, GD: Gender.”

\section{Discussions and Conclusions}

Service performance of employees is an essential source of economic improvement of a service institution as it helps to extricate one service institution over its competitors. Moreover, service workers of service industry are predictable to deliver service recovery performance (SRP) that helps in solving a facility failure and to gratify their clients by meeting their prospects. The study is, therefore, conducted on the mediating impact of EL on the relationship between TL and SRP. For this purpose, the primary data are gathered from 200 respondents currently working in service jobs that basically involve interaction of customers in Pakistan. Reliability test and Barron and Kenny (1986) method (through regression analyses) are used to analyze the mediating impact of EL on the relationship between TL and SRP.

Firstly, Nomological network of SRP has been added to the current study and the findings elaborates that SRP is positively affected by TL. These findings extend the previous research by analyzing the backgrounds of SRP because TL has associated with the performance of the employees who are working in service jobs (e.g., Lin, 2010b; Punjaisri et al., 2013), but this relation has not examined empirically. The findings of present study support that idea that TL work as motivation for employees for improving in their performance and the provision of greatest SRP in the situation of SR. So therefore, the conclusions provide support to the idea that TL is positively or directly related with SRP. Secondly, the present research also delivers a theoretical framework that links TL with SRP. 
Conclusions also elaborated the positive link of TL with DA and negative link with SA. Results also extend the previous studies on EL, as research shows that generally diverse graces of leadership effect the emotions of employees. The findings are similar with those studies which shows that TL motivates the workers tin order to support their performance with the objectives of an institution. In the framework of service jobs, an organization's objective must act deep to show the honest positive emotions instead of showing false emotions through SA. Findigs are also similar with those studies which concluded that TL provoke progressive emotions in employees (e.g., McCaell-Keaedy and Andaron, 2002; Walter and Brach, 2008).

This study also implies some practical consequences for the industry of services. Particularly there are two consequences through which SR management is directly affected by institutions. Firstly, the findings depict that SRP is directly or indirectly affected by DA correspondingly. These results support the privileges of previous literature that throughout the service interactions, clients can discriminate DA and SA. (Zho et al., 2014; Hung et al., 2016). Thus, there is need to make effort to increase DA over SA. DA is associated with understanding, sympathetic, and curiosity, central intellectual and attitudinal foundations of SRP and service recovery interactions are directly affected by these subordinate emotions. Hence, inspiring and teaching employees to participate in DA can help in enhancing the superiority of workers' SRP and eventually encourage these outcomes of effective service recovery. Secondly, SR of management performance is also affected by the styles of leadership. So, manager's preparation should focus on preparation managers to engage in performances of TL. TL are operative in encouraging the performance of followers by linking their individuality with the institution, by stimulating followers by being a role model, and considerate the assets and faintness of followers. Thus, TL could help assistants better recognize with objectives of institutions. Similarly, SA should decrease for increasing SRP through demonstrating DAEL strategies.

This study has few limitations. First, TL was measured from the viewpoint of the follower and could limit the findings of this study. As such, a multi-level examination of how TL effect SRP could be useful for future contributions. Second, the current paper did not include a formal test of the moderating effect of culture, which is an area for future research.

\section{References}

Babakus, E., Yavas, U., Karatepe, O.M., Avci, T., (2003). The effect of management commitment to service quality on employees' affective and performance outcomes. J. Acad. Mark. Sci. 31 (3), 272-286

Chuang, A., Judge, T. A., \& Liaw, Y. J. (2012). Transformational leadership and customer service: A moderated mediation model of negative affectivity and emotion regulation. European Journal of Work and Organizational Psychology, 21(1), 28-56.

Dasborough, M.T., (2006). Cognitive asymmetry in employee emotional reactions to leadership behaviors. Leadersh. Q. 17 (2), 163-178.

Dasborough, M.T., Ashkanasy, N.M., (2002). Emotion and attribution of intentionality in leader-member relationships. Leadersh. Q. 13 (5), 615-634.

Grandey, A.A., 2000. Emotional regulation in the workplace: a new way to conceptualize emotional labor. J. Occup. Health Psychol. 5 (1), 95.

Guchait, P., Paşamehmetoğlu, A., Dawson, M., (2014). Perceived supervisor and co-worker support for error management: impact on perceived psychological safety and service recovery performance. Int. J. Hosp. Manage. 41, 28-37.

Guchait, P., Simons, T., \& Pasamehmetoglu, A. (2016). Error recovery performance: the impact of leader behavioral integrity and job satisfaction. Cornell Hospitality Quarterly, 57(2), 150-161.

Ha, J., Jang, S.S., 2009. Perceived justice in service recovery and behavioral intentions: the role of relationship quality. Int. J. Hosp. Manage. 28 (3), 319-327.

Huang, P.F., Dai, C.W., (2010). The impacts of emotional contagion and emotional labor perception on employees' service performance. Int. J. Electron. Bus. Manage. 8 (1), 68.

Lee, Y.K., Son, M.H., Lee, D.J., (2011). Do emotions play a mediating role in the relationship between owner leadership styles and manager customer orientation, and performance in service environment? Int. J. Hosp. Manage. 30 (4), 942-952

Jiang, W., Zhao, X., \& Ni, J. (2017). The impact of transformational leadership on employee sustainable performance: The mediating role of organizational citizenship behavior. Sustainability, 9(9), 1567.

Kau, A.K., Wan-Yiun Loh, E., (2006). The effects of service recovery on consumer satisfaction: a comparison between complainants and non-complainants. J. Serv. Marketing 20 (2), 101-111.

Kim, H.J., (2008). Hotel service providers' emotional labor: the antecedents and effects on burnout. Int. J. Hosp. Manage. 27 (2), 151-161.

Kim,S.M., Oh,J.Y.,(2012). Employee emotional response toward health care organization's service recovery efforts and its influences on service recovery performance. Serv. Bus. 6 (3), 297-321.

Lee, M.J., Singh, N., Chan, E.S., (2011a). Service failures and recovery actions in the hotel industry: a text-mining 
approach. J. Vacat. Market. 17 (3), 197-207.

Liao, H., (2007). Do it right this time: the role of employee service recovery performance in customer-perceived justice and customer loyalty after service failures. J. Appl. Psychol. 92(2), 475.

Luo, A., Guchait, P., Lee, L., \& Madera, J. M. (2019). Transformational leadership and service recovery performance: The mediating effect of emotional labor and the influence of culture. International Journal of Hospitality Management, 77, 31-39.

Lyubomirsky, S., King, L., Diener, E., (2005). The benefits of frequent positive affect: does happiness lead to success? Psychol. Bull. 131 (6), 803.

Punjaisri, K., Evanschitzky, H., Rudd, J., (2013). Aligning employee service recovery performancewith brandvalues: the roleofbrand-specificleadership. J.Market.Manage. 29 (9-10), 981-1006. 